\title{
Myosteatosis: a relevant, yet poorly explored element of sarcopenia
}

\author{
Mauro Zamboni ${ }^{1} \cdot$ Stefano Gattazzo ${ }^{1} \cdot$ Andrea P. Rossi $^{1}$
}

Received: 31 October 2018 / Accepted: 13 November 2018 / Published online: 30 November 2018

(c) European Geriatric Medicine Society 2018

Great interest has been given to sarcopenia and its association with increased risk of morbidity and mortality. However, sarcopenia is now considered a muscle disease characterized by poor muscle function and quality [1]. Interrelationships between these components of sarcopenia have been not yet completely studied.

Quality of muscle, usually defined by the ratio between muscle strength and mass, may refer to muscle's micro- and macroscopic changes. Muscle composition may be affected by the deposition of fat between skeletal muscle bundles and beneath the muscle fascia, called intermuscular fat, and by fat deposition inside muscle fibers, called intramuscular fat $[2,3]$. Evaluation of fat deposition in muscles is complex, far from clinical practice, and may be obtained only using imaging techniques or more invasive methods like muscle biopsies. Computed tomography and magnetic resonance are considered the gold standard methods for intramuscular and intermuscular fat evaluation, respectively, while other body composition methods, such as DXA and BIA do not allow to evaluate muscle quality parameters.

It is well-known that fat deposition inside the muscle is strongly associated with obesity, diabetes and metabolic syndrome [2]. Both intra and intermuscular fat are higher in males compared to females and increase with age. In addition, there is a close link between fat deposition in muscles and other fat compartments, especially visceral fat and in general ectopic fat deposition (i.e., deposition of fat in liver, pancreas and heart). Moreover, intermuscular fat has been found to be a good predictor of insulin sensitivity. In fact, recent studies showed that the accumulation of fatty acids, LC-CoA, DAG, and ceramides decreases insulin action by

This comment refers to the article available at https://doi. org/10.1007/s41999-018-0110-y.

Mauro Zamboni

mauro.zamboni@univr.it

1 Geriatric Division, Department of Medicine, University of Verona, Verona, Italy inhibiting the insulin PI3 K/Akt/mTOR signaling pathway [4].

Fatty infiltration in the skeletal muscle may alter muscle fiber orientation and affect muscle strength, increasing the risk of mobility deficits onset. Further, intramuscular fat is a metabolically active component of muscle, able to secrete inflammatory cytokines leading to systemic inflammation, muscle cells proliferation and differentiation decline and also muscle catabolism.

Perkisas et al. [5] in their paper published in this edition of the European Geriatric Medicine Journal examined the relationship between intramuscular fat and the other components of sarcopenia in 302 older hospitalized patients. All the subjects enrolled in this study underwent evaluation of muscle mass and intramuscular fat, as assessed by mid-thigh computed tomography, and muscle strength by dynamometer and functional capacity by short physical performance battery. Further, Perkisas et al. [5] examined whether all the component of sarcopenia, in particular intramuscular fat, were associated with 4-year mortality in this cohort of subjects.

As expected, Perkisas et al. observed that intramuscular fat was negatively correlated with both muscle strength and function. Furthermore, a significant association between intramuscular fat and mortality was found in males aged 70-79 years, but not in the whole study sample; a clear association between muscle mass, strength and function and mortality was observed in all subjects confirming and expanding previous observations, such as that observed in a cohort of hospitalized geriatric patients [5-8].

Perkisas' results of an association between muscle fat infiltration and mortality are in line with those of Reinders et [9], observed in a wide study sample of men and women aged 76.4 years with a follow-up of 8.8 years, confirming that muscle quality is an important measure.

Intramuscular fat is an early architectural muscle change that occurs before the onset of strength and functional abnormalities as well as before the metabolic alterations linked with obesity and diabetes. Fat deposition inside the muscles, i.e., myosteatosis, together with myofibrosis, significantly 
reduces muscle quality, thus its association with morbidity and mortality is not surprising.

Nevertheless, two important messages should be taken home from the paper of Perkisas et al. [9]. (1) Myosteatosis should be addressed in the workup of sarcopenia, as it correlates with muscle strength and physical performance, as well as lower survival; (2) interventions aimed to prevent or delay disability and decline mortality in the older people should not focus only on increasing muscle strength, but also on optimizing muscle quality by preventing fat deposition inside muscles. Behavioral and pharmacological interventions should be performed to decrease fat infiltration in muscle, and thus to improve muscle strength and performance. Intervention programs including nutritional interventions associated with strength and exercise training, may decrease fat infiltration inside the muscle [10], improve muscle quality and function and as a consequence could delay physical disability and mortality in older people.

\section{Compliance with ethical standards}

Conflict of interest The authors declare that they have no conflict of interest.

Ethical approval This article does not contain any studies with human participants or animals performed by any of the authors.

Informed consent For this type of study formal consent is not required.

\section{References}

1. Cruz-Jentoft AJ et al (2018) Sarcopenia: revised European consensus on definition and diagnosis. Age Ageing 1-16

2. Hausman GJ et al (2014) Intermuscular and intramuscular adipose tissue: bad vs. good adipose tissues. Adipocyte 3(4):242-255

3. Goodpaster BH (2002) Measuring body fat distribution and content in humans. Curr Opin Clin Nutr Metab Care 5(5):481-487

4. Kelley DE (2000) Overview: what is insulin resistance? Nutr Rev 58(3):2-3

5. Perkisas $\mathrm{S}$ et al (2018) The relation between mortality, intramuscular adipose tissue and other components of sarcopenia in hospitalized geriatric patients. Eur Geriatr Med 9(6):801-807

6. Visser M et al (2005) Muscle mass, muscle strength, and muscle fat infiltration as predictors of incident mobility limitations in well-functioning older persons. J Gerontol 60(3):324-333

7. Nam $\mathrm{S}$ et al (2016) Lower body function as a predictor of mortality over 13 years of follow up: findings from Hispanic established population for the epidemiological study of the elderly. Geriatr Gerontol 16(12):1324-1331

8. Hirani V et al (2015) Sarcopenia is associated with incident disability, institutionalization, and mortality in community-dwelling older men: the concord health and ageing in men project. J Am Med Dir Assoc 16(7):607-613

9. Harris TB et al (2007) Age, gene/environment susceptibility (AGES)-Reykjavik study: multidisciplinary applied phenomenics. Am J Epidemiol 165(9):1076-1087

10. Goodpaster BH et al (2008) Effects of physical activity on strength and skeletal muscle fat infiltration in older adults. J Appl Physiol 105(5):1498-1503 